\title{
THERMAL GRADIENTS IN THE OUTER LUNAR LAYERS
}

\author{
J. A. BASTIN, S. J. PANDYA, and D. A. UPSON \\ Queen Mary College, University of London, England
}

\begin{abstract}
During the next Apollo mission Apollo 15, it is planned to fix thermocouples at various depths up to $3 \mathrm{~m}$ below the lunar surface. It seems likely that the resulting temperature measurements will show a positive temperature differential with depth resulting from a net outward heat flux. It is the purpose of this paper to examine experiments already carried out which indicate a temperature gradient. Since the thermal flux is of direct importance in fundamental problems of lunar origin and the nature of the lunar interior, the relation between this quantity and the temperature gradient will be examined.
\end{abstract}

\section{Thermal Balance in the Outer Surface Layers}

The thermal transfer relaxation time for a system of linear dimension $L$ is given by:

$$
\tau \sim L^{2}\left(\frac{\varrho c}{k}\right)
$$

in which $\varrho$ is the density, $c$ the specific heat and $k$ a thermal transfer coefficient which we can formally introduce to take into account the effects of convection, radiative transfer and true lattice conduction. It is well known that, if we introduce only thermal lattice conductivity into Equation (1) taking the values of diffusivity $k / \varrho c$ from the measurements of lunar basalts measured at $230 \mathrm{~K}$ (Horai et al., 1970) then Equation (1) shows that the thermal relaxation time $\tau$ for the Moon as a whole is greater than $t_{m}$, the age of the Moon, by a factor of about $10^{2}$. However, over smaller distances of the order of $50 \mathrm{~km}$ and less the reverse is the case and Equation (1) indicates that the heat flux over consecutive layers is virtually constant for all layers within this distance scale. In this case, if $r_{1}$ and $r_{2}$ are the radii, measured from the lunar centre, of two relatively close spheres, then

$$
\begin{aligned}
& r_{1}^{2} k_{1}\left(\frac{\partial T}{\partial r}\right)_{r=r_{1}}=r_{2}^{2} k_{2}\left(\frac{\partial T}{\partial r}\right)_{r=r_{2}} \\
& r_{1}-r_{2} \ll\left[\left(\frac{k}{\varrho c}\right) t_{n}\right]^{1 / 2} .
\end{aligned}
$$

We now apply Equation (2) to two layers, one in the regolith whose thermal gradient is to be measured by Apollo 15 and the second in the bedrock some kilometers below the lunar surface. The limit of sensitivity of a thermocouple measurement is of the order of $10^{-2} \mathrm{~K}$ and it is at once clear from Equation (2) that the Apollo 15 mission will only be able to measure the temperature gradient with worthwhile fractional accuracy if the regolith layer has a thermal conductivity which is very much less than that of the bedrock below. The conductivity of the fines (Cremers et al., 1970; Gough et al., 1971) measured in vacuo in the laboratory is nearly a factor of $10^{3}$ less than 
that for type A basalt samples (Horai et al., 1970). If we now, as a first approximation, identify type A basalt with the bedrock and the regolith with surface fines of constant density, we can determine the thermal gradient in the regolith providing we know the thermal gradient in the bedrock below. Estimates of this bedrock gradient may be obtained from evolutionary models (e.g., McDonald, (1959), $4 \times 10^{-3} \mathrm{~km}^{-1}$ ) but a more direct estimate comes from the magnetometer experiment $\left(2 \times 10^{-3} \mathrm{~K} \mathrm{~m}^{-1}\right.$; Sonett et al. (1971)). The two approaches agree within an order of magnitude, and together with laboratory rock measurements already quoted show that we should expect from Equation (2) a regolith gradient of $2 \mathrm{~K}$ per meter, a value which should be measureable with high accuracy by the Apollo 15 experiment.

Perhaps the most questionable assumption made in the above approximate treatment is the identification of the conductivity of the lunar surface layer with that of fines measured in the laboratory, and this will now be considered in detail.

\section{Thermal Conductivity of the Lunar Regolith Layers}

We first review briefly the direct thermal conductivity measurements made in the laboratory with lunar fines.

Perhaps the most reliable method is the line source method employed by Cremers et al. (1970 and 1971). The method uses a straight wire as a heat source within the powdered fines and the wire itself acts as its own resistance thermometer. The method shows the expected increase of effective conductivity with temperature resulting from the radiative contribution to the thermal transfer: although there is some experimental scatter, it seems unlikely that the measurements are more in fractional error than about 0.2 . The method gives a value of $1.9 \times 10^{-3} \mathrm{Wm}^{-1} \mathrm{~K}^{-1}$ at the mean lunar temperature $230 \mathrm{~K}$.

A more recent method reported by Gough et al. (1971) relies essentially on subjecting the base of a thin cylinder of fines to a step function change of temperature and measuring the radiation from the top surface as a function of time so as to determine the time variation of effective temperature of the top surface. The method is not so well developed as the line source method although it should be relatively accurate. At $320 \mathrm{~K}$ a value of $6.0 \times 10^{-3} \mathrm{Wm}^{-1} \mathrm{~K}^{-1}$ is obtained which, in view of the increase of conductivity with temperature, is in fair agreement with the line source method.

Whilst both the above methods probably give reasonably accurate values of the conductivity of the fines under the laboratory conditions, the measured values may for several reascns differ from those of the lunar surface layer. Firstly, in both cases the material was packed into a container so that the important intercrystalline contacts could well differ considerably from those of material on the lunar surface; secondly, although the measurements were made under high vacuum conditions, the samples had in both cases been exposed to air prior to the experiment. For these reasons it is of considerable interest that a laboratory measure of the far infrared absorption coefficient (Bastin et al., 1970; Ade et al., 1971) makes it possible to determine the 
conductivity of the top few centimeters of the lunar surface. Unlike the thermal conductivity the infrared absorption coefficient would be expected to be relatively insensitive to transportation and packaging. This measurement, together with microwave measurements at different frequencies of the lunar brightness temperature throughout a lunation, enable the quantity $k \varrho / c$ to be determined and this relates directly to the top few centimetres of the lunar surface since it is only this layer which contributes to the microwave measurements. Since $\varrho$ and $c$ are known with considerable accuracy, the method enables $k$ to be determined directly and the value obtained is considerably lower than that found from the direct laboratory determination $\left(10^{-4} \mathrm{Wm}^{-1} \mathrm{~K}^{-1}\right)$.

Although the top few centimeters of the lunar surface probably have very low conductivity, it is by no means sure that such conductivities extend into the regolith. Gough et al. (1971) have shown that compression of fines causes a considerable increase of conductivity and the various thermal and shock annealing processes known to exist in the regolith might well be expected to increase the conductivity still further - perhaps by an order of magnitude or more so that it approaches more the conductivity of the bedrock.

The experimental evidence for this comes from measurements of diffusivity of breccia $\left(5.85 \times 10^{-7} \mathrm{~m}^{2} \mathrm{~s}^{-1}\right)$ by Horai et al. (1970). Taking a value for density as $2.21 \times 10^{3} \mathrm{~kg} \mathrm{~m}^{-3}$ and for specific heat as $5.40 \times 10^{2} \mathrm{~J} \mathrm{~kg}^{-1} \mathrm{~K}^{-1}$ the thermal conductivity at the mean lunar temperature of about $200 \mathrm{~K}$ turns out to be $0.7 \mathrm{~W} \mathrm{~m}^{-1} \mathrm{~K}^{-1}$. This value is not appreciably less than the conductivity of basalt $\left(1.6 \mathrm{~W} \mathrm{~m}^{-1} \mathrm{~K}^{-1}\right)$ Horai et al. (1970).

Considering breccia to be made from compressed fines, the result shows a marked increase in conductivity as the inter-grain contact is increased either by pressure or by melting. This shows that the thermal gradient would rapidly approach the value of the bedrock as one goes deeper in the regolith.

In conclusion, from direct measurements on lunar samples, it would thus seem that the regolith layer may have a thermal differential over its depth of several degrees $\mathrm{K}$ per meter, if not more. In view of the relatively hard and stable nature of this layer, such a result may seem intuitively unlikely and we therefore will look at other experiments which also indicate an appreciable gradient in the regolith.

\section{Temperature Gradients in the Regolith}

Although in the proposed Apollo 15 experiment there may be some problems with ensuring that the thermocouple junctions are strictly at the temperature of the nearby rock, there is no doubt that the experiment should be very reliable. It is, however, of interest at this time to consider the other methods by which this gradient has been inferred.

The first method utilises measurements of the lunar brightness temperature $T_{\lambda}$ at wavelengths in the range $0.05-0.50 \mathrm{~m}$. Throughout this range lunar rock has a relatively low electromagnetic absorption coefficient so that radiation received at the Earth comes not from the surface but is a sum of contributions from depths ranging up to 
many metres below the surface. If $T$ is the thermodynamic temperature at depth $z$ then it follows from the theory of radiative transfer that for the center of the Moon's disc

$$
T_{\lambda}=\left(1-R_{0}\right) \int_{z=0}^{\infty} k_{\lambda} \varrho T_{z} e^{-k_{\lambda} \varrho z} \mathrm{~d} z
$$

where $k_{\lambda}$ and $\varrho$ are the electromagnetic mass attenuation coefficient and density respectively. $R_{0}$ is the reflection coefficient at normal incidence. If now we assume a uniform temperature gradient $b$ with $T_{0}$ as the surface temperature

$$
T_{z}=T_{0}+b z
$$

then Equation (3) gives the measured brightness temperature

$$
T_{\lambda}=\left(1-R_{0}\right)\left[T_{0}+\frac{b}{k_{\lambda} \varrho}\right] .
$$

Radar reflection measurements and measurements on lunar samples show that $\left(1-R_{0}\right)$ lies between 0.90 and 0.97 and does not vary appreciably with wavelength. The differential of Equation (5) with $\lambda$ would therefore be expected to give a direct measure of the thermal gradient which can be found absolutely if $k_{\lambda}$ and $\varrho$ are known. A discussion by Ade et al. (1971) indicates that

$$
k_{\lambda \varrho}^{1}=2 \cdot 6 \lambda
$$

so that (5) and (6) give

$$
\frac{\partial T_{\lambda}}{\partial \lambda}=2 \cdot 3 b .
$$

(It will be noted that the factor on the left hand side of (7) is dimensionless, i.e. it has the same numerical value whatever units the other quantities are expressed in. This would be expected since both $b$ and $\partial T_{\lambda} / \partial \lambda$ have the dimensions of temperature divided by distance.)

The result given in Equation (7) strictly refers only to the center of the lunar disc. A more detailed treatment following a different approach but averaging over the whole disc has been given by Krotikov and Troitski (1964). The results of this analysis only modify Equation (7) by relatively small factors. The absorption coefficients for the lunar fines (Ade et al., 1971) show the absorption coefficient of the lunar rock at $2 \mathrm{~mm}$ wavelength to be greater than anticipated before the return of rock samples.

To some extent this discrepancy has been removed by the discovery of a scattering component in the fines (Clegg et al., 1972).

If we take the values of the brightness temperature measured as a function of wavelength collected both by Troitskii (1967) and Linsky (1966) values of about $35 \mathrm{~K} \mathrm{~m}^{-1}$ are obtained for the thermal gradient.

The thermoluminescence data taken from various depths in the core samples (Hoyt 
et al., 1971) also give evidence of a strong thermal gradient. The rising curve in the first few centimeters is without doubt the effect of high temperature on the top part of the surface due to the diurnal thermal input. From 8-40 cm, however, superimposed on random fluctuations, there is a marked slope which would be accounted for by a positive temperature gradient.

When allowance is made for the variation of ionisation with depth the method gives a value of $2 \pm 2 \mathrm{~K} \mathrm{~m}^{-1}$ for the gradient.

All the work cited so far has shown a positive temperature differential with depth. However, a recent publication by Salisbury and Fernald (1971) using Arecibo Radio Telescope in the metre wavelength range indicates an inverse temperature gradient with depth. The reason given for this effect was the transport of heat by latent heat changes during the evaporation of mercury.

Our preliminary calculations based on the available data of the mercury content of the lunar soil gives a very negligible gradient due to this effect and we believe a probable error in telescope calibration could have caused this effect.

Since the writing of this paper, Apollo 15 experiment (Langseth, 1972) has shown a gradient of $1.75 \mathrm{~K} \mathrm{~m}^{-1}$ at depth below which thermal wave has negligible effect. The experiment also measured the conductivity at depths of the order of 1 metre giving a value of $1.7 \times 10^{-2} \mathrm{~W} \mathrm{~m}^{-1} \mathrm{~K}^{-1}$. These two results together with the work of Sonett et al. (1971) imply a surprisingly high conductivity for the deep bedrock.

\section{References}

Ade, P. A., Bastin, J. A., Marston, A. C., Pandya, S. J., and Puplett, E.: 1971, Proc. Second Lunar Science Conference 3, 2203.

Bastin, J. A., Clegg, P. E., and Fielder, G.: 1970, Proc. Apollo XI Lunar Science Conference 3, 1987.

Clegg, P. E., Pandya, S. J., Bastin, J. A., and Foster, S.: 1972, 'Far Infrared Properties of Lunar Rock', Proc. Third Lunar Science Conference, to be published.

Cremers, C. J., Birkebak, R. C., and Dawson, J. P.: 1970, Proc. Apollo XI Lunar Science Conference 3, 2045.

Cremers, C. J. and Birkebak, R. C.: 1971, Proc. Second Lunar Science Conference 3, 2311.

Gough, G. O., Pandya, S. J., and Bastin, J. A.: 1969, Private Communication.

Horai, K., Simmons, G., Kanamori, H., and Wones, D.: 1970, Proc. Apollo XI Lunar Science Conference 3, 2243.

Hoyt, H., Jr., Miyajima, M., Walker, R., Zimmerman, D., Zimmerman, J., Britton, D., and Kardos, J. L.: 1971, 'Radiation Dose Rates and Thermal Gradients in the Lunar Regolith: Thermoluminescence and DTA of Apollo XII Samples' 3, 2245.

Krotikov, V. D. and Troitskii, V. S.: 1963, Soviet Phys. Uspekhi 6, 841.

Langseth, M. G. Jr.: 1972, Third Lunar Science Conference 3, 475.

Linsky, J. L.: 1966, Icarus 5, 606.

MacDonald, G. J. F.: 1959, J. Geophys. Res. 64, 1967.

Sonett, C. P., Colburn, D. S., Dyal, P., Parkin, C. W., Smith, B. F., Schubert, G., and Schwartz, K.: 1971, Nature 230, 359. 